\title{
Direct Observation of the Transition from Free to Constrained Single-Segment Motion in Entangled Polymer Melts
}

\author{
A. Wischnewski, M. Monkenbusch, L. Willner, and D. Richter \\ Institut für Festkörperforschung, Forschungszentrum Jülich, D-52425 Jülich, Germany \\ G. Kali \\ Institut Laue-Langevin, B.P. 156X, Avenue des Martyrs, F-38042 Grenoble Cedex 9, France
}

(Received 14 August 2002; published 6 February 2003)

\begin{abstract}
We report a direct determination of the time dependent mean-squared segment displacement of a polymer chain in the melt covering the transition from free to constraint Rouse relaxation along the virtual tube of the reptation model. This has been achieved by a neutron spin-echo (NSE) measurement of the segmental self-correlation function as conveyed by the spin-incoherent scattering from two fully protonated polymer melts, polyethylene and polyethylene propylene. Within the scenario of de Gennes reptation model a transition of the time dependence of segmental mean-squared displacements from $\propto t^{1 / 2}$ to $\propto t^{1 / 4}$ is expected and clearly corroborated by the incoherent NSE results.
\end{abstract}

DOI: $10.1103 /$ PhysRevLett.90.058302

PACS numbers: 47.50.+d, 83.10.Kn

Beyond a certain length polymer chains in a melt are subject to topological constraints of motion due to entanglements. As a consequence in high molecular weight (M) melts the viscosity $\eta$ becomes proportional to $M^{3.4}$ instead of $\eta \propto M$ for low molecular weights [1]. Furthermore in the viscoelastic properties of the melt at intermediate frequency $\omega$ a plateau in the modulus $G(\omega)$, i.e., a transient rubbery network is observed [2]. A comprehensive recent review on viscoelasticity in entangled polymers may be found in Ref. [3]. Microscopically the single chain structure factor $S(Q, t)$ as observed by neutron spin-echo (NSE) spectroscopy on deuterated melts containing a few $h$-labeled chains provides clear evidence for the restriction of motion [4,5] and corrobates all the features of the de Gennes dynamic structure factor for local reptation [6-9]. The mean-squared segmental displacements $\left\langle r^{2}(t)\right\rangle$ in the reptation scenario are given by an initial entropy driven Rouse motion $\left[\left\langle r^{2}(t)\right\rangle \propto t^{1 / 2}\right]$ for $t<\tau_{e}$. At $\tau_{e}$ the segmental diffusion slows down due to the "contact" with the "walls" of the virtual tube, further motion, termed local reptation, is a quasi-onedimensional Rouse relaxation along the contour of the virtual tube $\left[\left\langle r^{2}(t)\right\rangle \propto t^{1 / 4}\right]$ [8]. This work presents the first direct measurements of $\left\langle r^{2}(t)\right\rangle$ in space and time in terms of the proton self-correlation function of diffusing segments in long chain polyethylene (PE) and polyethylene propylene (PEP) melts. The measured self-correlation function very clearly displays the predicted crossover from free Rouse motion to local reptation. Considering the non-Gaussian character of the segment motion along a 1D contorted tube, in the frame of the reptation model the single chain dynamic structure factor and the selfcorrelation function agree quantitatively. The resulting tube diameter thereby is significantly larger than what is inferred from rheology $[10,11]$.
The PE and PEP samples were obtained from parent 1,4-polybutadiene and polyisoprene, respectively, which were synthesized by anionic polymerization [12]. PE and PEP were obtained by subsequent hydrogenation (deuteration). For the present investigations $\mathrm{PE}$ with a molecular weight $M_{w}=190 \mathrm{~kg} / \mathrm{mol}$ and PEP with $M_{w}=$ $80 \mathrm{~kg} / \mathrm{mol}$ was chosen, both well in the entangled state.

The incoherent NSE experiments have been performed at the NSE-FRJ2 spectrometer at the DIDO reactor in Jülich [13] on fully protonated PE and PEP samples of $0.3 \ldots 0.4 \mathrm{~mm}$ thickness. The polymer layers had a transmission of $60 \%$. There are some disadvantages as well as virtues of using a conventional NSE spectrometer for the spectroscopy of spin-incoherent scattering. First of all, NSE is the only neutron spectroscopic method with sufficient time/energy resolution in the relevant $Q$ range, but there is an inherent penalty due to the fact that the spin of the scattered neutron is flipped with a probability of $2 / 3$, i.e., the remaining net polarization is $-1 / 3$ of the original yielding a sign reversed reduced echo signal on an additional background amounting to $2 / 3$ of the total scattering. A virtue of the spin-flip scattering is that multiple scattering is efficiently suppressed since two subsequent scattering events only leave $1 / 9$ of the signal and more events further reduce the contribution to the echo amplitude. The overall intensity is much smaller $(10 \ldots 20$ times) than the coherent small angle scattering from chain labeled samples. Because of the low intensity very careful background measurements from the sample container and spectrometer components are necessary. The total measuring time for each single $(Q, t)$ pair (including background) was $5 \mathrm{~h}$, requiring for a very high temporal stability of the instrument. The neutron wavelength of $\lambda=0.8 \mathrm{~nm}$ allowed for a Fourier time range $0.1 \mathrm{~ns} \leq \mathrm{t} \leq 22 \mathrm{~ns}$ in the normal instrument setup and 
$0.01 \mathrm{~ns} \leq \mathrm{t} \leq 0.13 \mathrm{~ns}$ in a special short time configuration with small precession coils in the sample region. All data shown stem from an integrated detector area of $615 \mathrm{~cm}^{2}$.

Figure 1 shows the incoherent scattering data from the hPE sample at $509 \mathrm{~K}$. The background has been subtracted as measured using the measured transmission ratio, without any further corrections. Note that the short time behavior follows the Rouse prediction (solid lines), i.e., without further correction $S_{\text {inc }}(Q, t \rightarrow 0) \rightarrow 1$ as expected.

For the very long PE and PEP chains studied here virtually all of the scattering intensity stems from "inner" segments. These should exhibit identical segmental diffusion and if the assumption of a Gaussian shape of the diffusive displacement probability distribution would hold for all times the scattering function could be written as

$$
S_{\text {inc }}(Q, t)=\exp \left[-\frac{1}{6} Q^{2}\left\langle r^{2}(t)\right\rangle\right]
$$

allowing to extract the mean-squared segment displacement directly from $-6 \ln \left[S_{\text {inc }}(Q, t)\right] / Q^{2}=\left\langle r^{2}(t)\right\rangle$. Figure 2 displays the hPE data in this representation.

As predicted by the standard Rouse theory [8] as well as by a number of computer simulations [14-17] segment diffusion is described by the mean-squared displacement [18]

$$
\left\langle r^{2}(t)\right\rangle=2 \sqrt{W l^{4} / \pi} t^{1 / 2} .
$$

Inserting the previously determined value for the Rouse rate $W(509 \mathrm{~K}) l^{4}=7 \pm 0.7 \mathrm{~nm}^{4} / \mathrm{ns}$ [19] from the analysis of the single chain structure factor of low molecular PE melts, Eq. (2) is quantitatively corroborated. Also a transition to a regime with a different power law

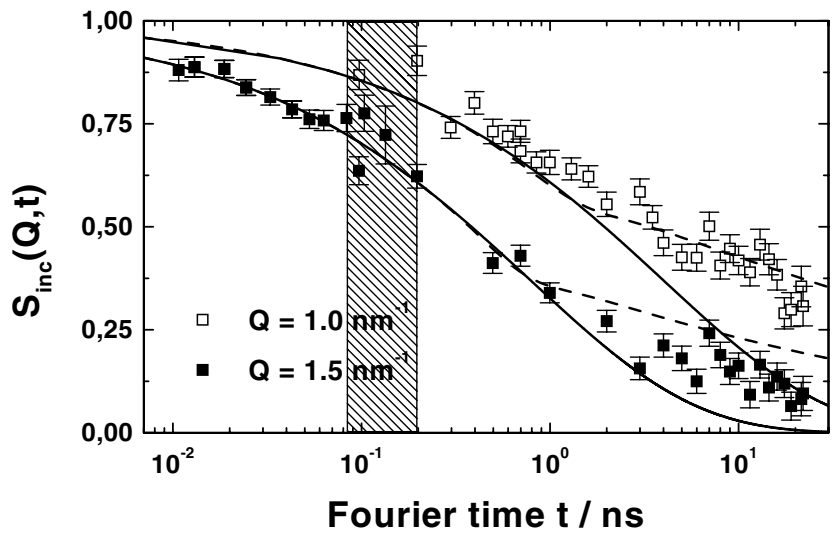

FIG. 1. NSE data obtained from the incoherent scattering from a fully protonated PE melt. At the range boundaries (gray bar) of the two spectrometer configurations (short or normal, see text) the data quality is worse than the bulk of the data points as seen by the sizes of the error bars. Lines see text. compatible with $\propto t^{1 / 4}$ is clearly visible. However, the crossover occurs at about $\tau_{e} \simeq 1 \mathrm{~ns}$ which is much less than the textbook value [8] derived as $\left[\tau_{e}\right]_{\mathrm{DE}}=d^{4} \pi /$ $\left(36 W l^{4}\right) \simeq 7 \mathrm{~ns}$, where the elementary step length of the Gaussion contorted virtual tube is identified with the tube diameter $(d=4.8 \mathrm{~nm}[7,9])$. Following Refs. $[8,18]$ the mean-squared segment displacement due to local reptation has been approximated as

$$
\left\langle r^{2}(t)\right\rangle^{\text {locRep }}=d \sqrt{2 / 3}\left(W l^{4} t / \pi\right)^{1 / 4} .
$$

Inserting $d=4.8 \mathrm{~nm}$ leads to the dotted line in Fig. 2, its intersection with the $\propto t^{1 / 2}$ line defines $\tau_{e}=\left[\tau_{e}\right]_{\mathrm{DE}}$, however the value of $7 \mathrm{~ns}$ is grossly wrong; fitting the data with $d$ as a free parameter rather yields $d=3 \mathrm{~nm}$.

Applying the same evaluation procedure as described above, the mean-squared displacements of $h$-PEP were obtained. Figure 3 displays the results.

Again a deviation from $\left\langle r^{2}(t)\right\rangle \propto t^{1 / 2}$ is clearly visible for $t \gtrsim 10 \mathrm{~ns}$ and Eq. (2) is corroborated for $t \lesssim 10 \mathrm{~ns}$. Using $W(492 K) l^{4}=3.26 \mathrm{~nm}^{4} / \mathrm{ns}[19]$, the data were fitted with Eqs. (2) and (3), yielding a crossover time of $\tau_{e}=8.4 \mathrm{~ns}$ and a tube diameter of about $4.3 \mathrm{~nm}$. To compare this result with the tube diameter obtained from the single chain dynamic structure factor of PEP, a measurement on a sample with $10 \%$ protonated PEP in a matrix of deuterated PEP has been performed at the ultrahigh resolution spin-echo spectrometer IN15 in Grenoble, France.

Figure 4 shows the result. The coherent dynamic structure factor in the framework of the reptation model is given by the expression of de Gennes [6]

$$
\begin{aligned}
\frac{S(Q, t)}{S(Q)}= & {[1-F(Q)] \exp \left(\frac{t}{\tau_{0}}\right) \operatorname{erfc}\left(\sqrt{t / \tau_{0}}\right) } \\
& +F(Q) S_{\text {escape }}\left(\tau_{d} ; Q, t\right),
\end{aligned}
$$

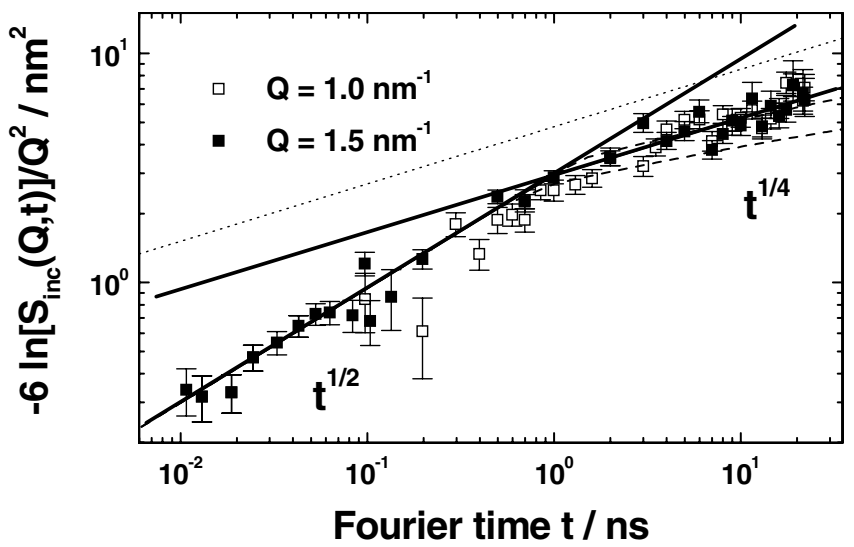

FIG. 2. Same data as shown in Fig. 1 in a representation of $-6 \ln \left[S_{\text {inc }}(Q, t)\right] / Q^{2}$, i.e., the mean squared displacement $\left\langle r^{2}(t)\right\rangle$ as long as the Gaussian approximation holds. Solid lines describe the asymptotic power laws $\left\langle r^{2}(t)\right\rangle \propto t^{1 / 2}, t^{1 / 4}$. Dashed and dotted lines: see text. 


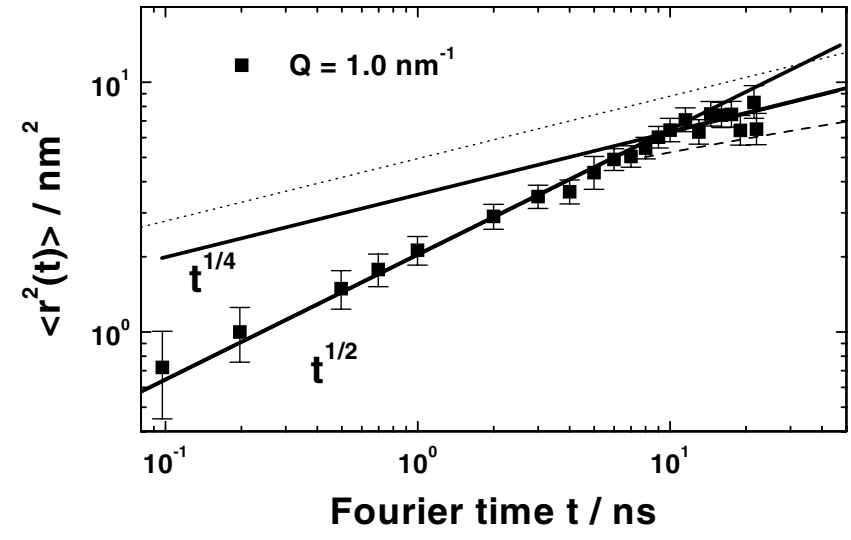

FIG. 3. Data of $h$-PEP in the representation of $-6 \ln \left[S_{\text {inc }}(Q, t)\right] / Q^{2}$ vs time for $T=492 \mathrm{~K}$. Solid lines describe the asymptotic power laws. Dotted and dashed lines: see text.

where $F(Q)=\exp \left[-(Q d / 6)^{2}\right]$ is the (cross-sectional) form factor of the tube. The two time scales in Eq. (4) are given by $\tau_{0}=36 /\left(W l^{4} Q^{4}\right)$ for the local reptation due to Rouse type segment diffusion along the tube and $\tau_{d}=$ $3 N^{3} l^{2} /\left(\pi^{2} W d^{2}\right)$ for the reptation type escape of the chain from the tube. Here, $N \propto M_{w}$ is the number of segments per chain $(N \simeq 2860)$. In the accessible NSE time range the tube escape term is virtually constant for the high $M_{w}$ PEP. The solid lines in Fig. 4 represent a fit with Eq. (4) simultaneoulsy to all $Q$ values. The tube diameter $d$ was varied as the only free parameter, yielding $d=6 \mathrm{~nm}$, i.e., $\left[\tau_{e}\right]_{\mathrm{DE}}=40 \mathrm{~ns}$ (see dotted line in Fig. 3), again in contradiction to $d=4.3 \mathrm{~nm}$ and $\tau_{e}=8.4 \mathrm{~ns}$, obtained from an interpretation of the incoherent data in terms of the Gaussian approximation.

In the derivation of Eq. (3) the Gaussian width after a diffusion time $t$ of the single segment distribution along

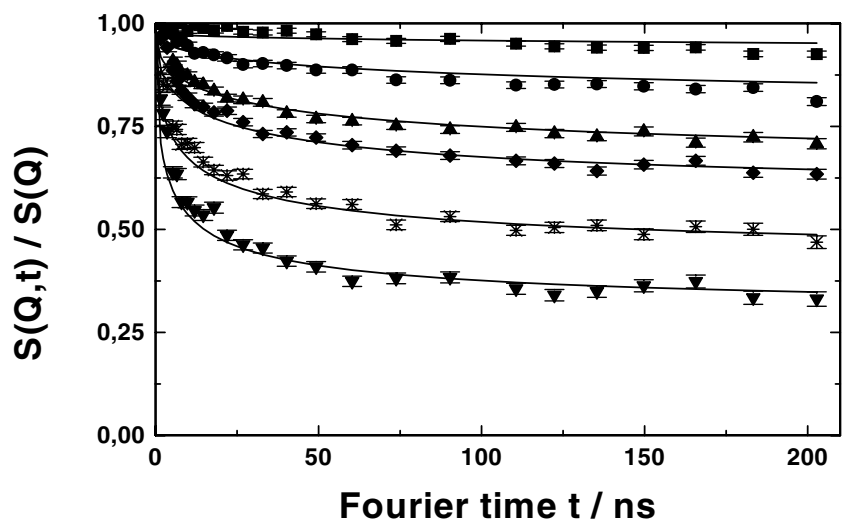

FIG. 4. Single chain dynamic structure factor $S(Q, t) / S(Q)$ from a $M_{w}=200 \mathrm{~kg} / \mathrm{mol}$ PEP melt at $T=492 \mathrm{~K}$ for the scattering wave vectors: $Q=0.3 \mathrm{~nm}^{-1}, Q=0.5 \mathrm{~nm}^{-1}, Q=$ $0.68 \mathrm{~nm}^{-1}, Q=0.77 \mathrm{~nm}^{-1}, Q=0.9 \mathrm{~nm}^{-1}, Q=1.15 \mathrm{~nm}^{-1}$, from above. Solid lines represent a fit with Eq. (4). the 1D tube contour path coordinate, $s$, is taken for the time dependent displacement. Projecting this on the Gaussian contorted tube again corresponds to a Gaussian sublinear diffusion in real space [Eq. (3)]. However, the real process has to be modeled by projecting the segment probability distribution due to curvilinear Rouse motion on the linear coordinate $s$ onto the random walk like contour path of the contorted tube leading to a non-Gaussian probability distribution of the segment at times $t>\tau_{e}$. The necessity to perform the proper averaging has first been shown by Fatkullin and Kimmich [20] in the context of interpretation of field-gradient NMR diffusometry data [21] which yield results that are analogous to the incoherent neutron scattering functions, however in another time and space regime covering mainly the regime $\tau_{R}<t<\tau_{d}$. Their result

$$
S_{\mathrm{inc}}\left(Q, t>\tau_{e}\right)=\exp \left[\frac{Q^{4} d^{2}}{72} \frac{\left\langle r^{2}(t)\right\rangle}{3}\right] \operatorname{erfc}\left[\frac{Q^{2} d}{6 \sqrt{2}} \sqrt{\frac{\left\langle r^{2}(t)\right\rangle}{3}}\right]
$$

invalidates the Gaussian approximation for times above $\tau_{e}$. We note that Eq. (5) is strictly valid only for $t \gg \tau_{e}$ when $\left\langle r^{2}(t)\right\rangle \gg d^{2}$. The effect on the scattering function is that if (wrongly) interpreted in terms of the Gaussian approximation the crossover to local reptation appears to occur at significant lower values of $\tau_{e}$.

Figsures 1-3, show a comparison of the scattering function $S_{\text {inc }}(Q, t)$ as predicted by Eqs. (1) and (5) (dashed lines) with the incoherent NSE data. The parameters $W l^{4}$ and $d$ were fixed to the values taken from the coherent single chain structure factor measurements. For PE and $Q=1 \mathrm{~nm}^{-1}$ the free Rouse regime $\left(t<\tau_{e}\right)$ as well as the local reptation regime is well reproduced by a combination of Eqs. (1) and (5). For $Q=1.5 \mathrm{~nm}^{-1}$ in the case of PE and for $Q=1 \mathrm{~nm}^{-1}$ for PEP the prediction of Eq. (5) lies slightly outside the error band of the data points (lower dashed line). With increasing $Q$ values the spatial resolution increases. Agreement with theory may be expected only for $Q$ values $<2 \pi / d$. For the tube diameter of $4.8 \mathrm{~nm}$ in PE the "limiting" wave vector would be $1.3 \mathrm{~nm}^{-1}$ which may explain why at $Q=1.5 \mathrm{~nm}^{-1}$ deviations become visible. The same holds for PEP, where with $d=6 \mathrm{~nm}$, the typical wave vector would be about $Q=$ $1 \mathrm{~nm}^{-1}$. Since Eq. (5) is strictly valid only when the segment displacement is large compared to the tube diameter, in the transition regime an unknown crossover function may modify its detailed behavior. Thus, whereas some questions remain on the validity of the limiting expression for $S_{\text {inc }}(Q, t)$ in the transition regime around $\tau_{e}$, deviations from the Gaussian approximation well above $\tau_{e}$ must be assumed. Besides the theoretical argument [20] the observed value of the crossover time $\tau_{e}$ provides strong evidence for this.

The above described behavior renders a direct comparison with published computer simulation results 
$[14,16,22,23]$ difficult as soon as the pure Rouse regime is left, since these are expressed in terms of mean-squared displacements/distances of various chain elements as a function of time. The simulations generally show a less pronounced transition from the $t^{1 / 2}$ to the $t^{p \simeq 1 / 4}$ regime than the present experimental results. The inferred exponent $p$ is somewhat larger than $1 / 4$, e.g., $(p \simeq 0.36)$ [16], or approaches $1 / 4$ [23]. To improve the comparison of incoherent scattering with the dynamic structure factor and computer simulation, $S_{\text {inc }}(Q, t)$ should be extracted from simulation results - with negligible extra effortwhere often only the mean-squared displacements are computed. These results could then be immediately compared with the neutron spectroscopic data in the short time range and versus field-gradient NMR at longer times. Finally, we note that the dimensions of local confinement observed at the proper mesoscopic scales are significantly larger than what is deduced from rheological tube diameter determination: $d^{\mathrm{PE}}=4.8 \mathrm{~nm}$ vs $d^{\mathrm{PE}}=3.2 \mathrm{~nm}$ (rheology) [10] and $d^{\mathrm{PEP}}=6 \mathrm{~nm}$ vs $d^{\mathrm{PEP}}=4.3 \mathrm{~nm}$ (rheology) [11]. Obviously the local freedom for segmental motion is larger than anticipated so far from rheology.

In conclusion incoherent neutron spin-echo spectroscopy has for the first time enabled a direct measurement of the crossover from single segment Rouse motion to local reptation. Applying the proper corrections for nonGaussianity effects the self-motion of a segment quantitatively agrees with the expectation of a tube model with parameters as obtained from the coherent single chain structure factor [9].

[1] G. Berry and T. Fox, Adv. Polym. Sci. 5, 261 (1968).

[2] S. Onogi, T. Masuda, and K. Kitagawa, Macromolecules 3, 109 (1970).

[3] H. Watanabe, Prog. Polym. Sci. 24, 1253 (1999).

[4] D. Richter, B. Ewen, B. Farago, and T. Wagner, Phys. Rev. Lett. 62, 2140 (1989).
[5] D. Richter, B. Farago, L. Fetters, J. Huang, B. Ewen, and C. Lartigue, Phys. Rev. Lett. 64, 1389 (1990).

[6] P. de Gennes, J. Phys. (France) 42, 735 (1981).

[7] P. Schleger, B. Farago, C. Lartigue, A. Kollmar, and D. Richter, Phys. Rev. Lett. 81, 124 (1998).

[8] M. Doi and S. Edwards, The Theory of Polymer Dynamics, International Series of Monographs on Physics Vol. 73 (Oxford University Press, Oxford, 1994).

[9] A. Wischnewski, M. Monkenbusch, L. Willner, D. Richter, A. E. Likhtman, T.C. B. McLeish, and B. Farago, Phys. Rev. Lett. 88, 058301 (2002).

[10] J. M. Carella, W.W. Graessley, and L. J. Fetters, Macromolecules 17, 2775 (1984).

[11] J. T. Gotro and W.W. Graessley, Macromolecules 17, 2767 (1984).

[12] M. Morton and L. J. Fetters, Rubber Chem. Technol. 48, 359 (1975).

[13] M. Monkenbusch, R. Schaetzler, and D. Richter, Nucl. Instrum. Methods Phys. Res., Sect. A 399, 301 (1997).

[14] T. Kreer, J. Baschnagel, M. Müller, and K. Binder, Macromolecules 34, 1105 (2001).

[15] R. Faller and F. Müller-Plathe, Chem. Phys. Chem. 2, 180 (2001).

[16] S. Kreitmeier, M. Wittkop, H. Trautenberg, T. Holzl, and D. Goritz, J. Comput. Phys. 133, 181 (1997).

[17] A. Kopf, B. Dunweg, and W. Paul, J. Chem. Phys. 107, 6945 (1997).

[18] The prefactor for $t^{1 / 2}$ behavior of the mean-squared displacement of inner segments $\left\langle r^{2}(t)\right\rangle$ within the Rouse model given in 6.4.4 of Doi and Edwards's textbook is not correct, the exact value is quoted in the text, it still may differ within a $10 \%$ band from direct summation results.

[19] D. Richter, B. Farago, R. Butera, L. Fetters, J. Huang, and B. Ewen, Macromolecules 26, 795 (1993).

[20] N. Fatkullin and R. Kimmich, Phys. Rev. E 52, 3273 (1995).

[21] E. Fischer, R. Kimmich, U. Beginn, M. Moller, and N. Fatkullin, Phys. Rev. E 59, 4079 (1999).

[22] W. Paul, K. Binder, D. Heermann, and K. Kremer, J. Chem. Phys. 95, 7726 (1991).

[23] K. Kremer, G. Grest, and I. Carmesin, Phys. Rev. Lett. 61, 566 (1988). 